\title{
Полупроводниковые гетероструктуры первого рода с непрямой запрещенной зоной на подложках с ориентацией (110)
}

\author{
(C) Д.С. Абрамкин ${ }^{1,2}$, Т.С. Шамирзаев ${ }^{1,2,3}$ \\ ${ }^{1}$ Институт фризики полупроводников им. А.В. Ржанова Сибирского отделения Российской академии наук, \\ 630090 Новосибирск, Россия \\ ${ }^{2}$ Новосибирский государственный университет, \\ 630090 Новосибирск, Россия \\ ${ }^{3}$ Уральский федеральный университет, \\ 620002 Екатеринбург, Россия \\ E-mail: demid@isp.nsc.ru
}

(Поступила в Редакцию 7 ноября 2018 г. В окончательной редакции 10 декабря 2018 г. Принята к публикации 17 декабря 2018 г.)

Гетероструктуры первого рода с непрямой запрещенной зоной являются удобными объектами для исследования спиновой динамики локализованных экситонов, изучение которой в других типах гетероструктур затруднительно. В работе показано, что структуры с таким энергетическим спектром могут быть сформированы из бинарных соединений $\mathrm{A}^{\mathrm{III}} \mathrm{B}^{\mathrm{V}}$ на подложках ориентации (110). Обсуждается влияние распределения механических напряжений и структуры зоны проводимости в пространстве квазиимпульсов на энергетический спектр электронных состояний в гетероструктурах.

DOI: 10.21883/FTP.2019.05.47569.9018

\section{1. Введение}

Применение низкоразмерных гетероструктур в оптоэлектронике дает возможность создания объектов с принципиально новыми свойствами, не характерными для объемных материалов. Это обусловлено существенным различием энергетического строения низкоразмерных полупроводниковых гетероструктур и объемных полупроводников [1]. Изменения энергетического спектра и симметрии электронных состояний при переходе от объемных материалов к гетероструктурам определяются эффектами размерного квантования, а также смещением краев энергетических зон вследствие упругих деформаций [2], вызванных рассогласованием параметров решеток материалов.

В настоящее время гетероструктуры на основе соединений $\mathrm{A}^{\mathrm{III}} \mathrm{B}^{\mathrm{V}}$ выращиваются главным образом на подложках с кристаллографической ориентацией (001). Между тем изменение ориентации подложки дает дополнительные возможности для управления параметрами структур. Так, например, в квантовых ямах (КЯ), выращенных на подложках ориентации (111), взаимная компенсация спин-орибитального взаимодействия, обусловленного эффектами Дрессельхауса и Рашбы, позволяет увеличивать длину диффузии спин-поляризованных электронов во внешнем электрическом поле $[3,4]$. Квантовые точки (KT) InAs/GaAs, сформированные на подложках ориентации (111), обладают симметрией $C_{3 v}$, обеспечивающей практически нулевую величину анизотропного обменного расщепления экситонных уровней [5], перспективны для создания источников запутанных фотонных пар [6].

В гетероструктурах с симметричными КЯ, выращенными на подложках с ориентацией (110), эффективное магнитное поле, обусловленное спин-орбитальным взаимодействием Дрессельхауза, направлено перпендикулярно плоскости КЯ $[7,8]$, что приводит к подавлению механизма спиновой релаксации Дьяконова-Переля для носителей заряда, спин которых направлен по оси роста гетероструктуры, и, как следствие, появлению устойчивых спиновых состояний $[7,9,10]$. Таким образом, использование гетероструктур, выращенных на подложках ориентации (110), открывает широкие возможности управления спиновой степенью свободы носителей заряда.

В отличие от электронов (дырок) для локализованных в гетероструктурах экситонов время жизни спина ограничено процессами рекомбинации. Поэтому для получения долгоживущих спин-поляризованных экситонов особый интерес представляют гетероструктуры, в которых скорость рекомбинации экситонов ограничена. Характерным примером таких объектов являются КЯ и КТ первого рода с непрямой запрещенной зоной. Разделение носителей заряда в пространстве квазиимпульсов в таких структурах приводит к увеличению времени излучательной рекомбинации экситонов до сотен микросекунд [11,12], что почти на 5 порядков величины превосходит время жизни экситонов в прямозонных системах [13].

Существование гетероструктур первого рода с непрямой запрещенной зоной, выращенных на подложках с ориентацией (001), было показано как теоретически $[14,15]$, так и экспериментально [16-25]. В то же время в литературе практически отсутствуют данные о гетероструктурах, сформированных на подложках с ориентацией (110).

В настоящей работе обсуждается возможность формирования гетероструктур первого рода с непрямой запрещенной зоной на основе соединений $\mathrm{A}^{\mathrm{III}} \mathrm{B}^{\mathrm{V}}$, вы- 
ращенных на подложках с ориентацией (110). Впервые проведены расчеты энергетических диаграмм псевдоморфно напряженных квантовых ям, сформированных из различных пар бинарных соединений $\mathrm{A}^{\mathrm{III}} \mathrm{B}^{\mathrm{V}}-$ фосфидов, арсенидов и антимонидов алюминия, галлия, индия с кубической симметрией элементарной ячейки (решетка сфалерита). Результаты расчетов могут быть использованы и для оценки энергетического спектра самоорганизующихся КТ, получаемых эпитаксиальными методами по механизму Странского-Крастанова, поскольку форма этих КТ такова, что основной вклад в размерное квантование носителей заряда вносит пространственное ограничение вдоль одной координаты вдоль направления роста [26].

\section{2. Методика расчета}

При расчете энергетического строения псевдоморфнонапряженных гетероструктур необходимо учитывать влияние деформаций, возникающих из-за рассогласования постоянных решеток материалов, на положения энергетических зон. Влияние деформаций на энергетическую структуру материалов выражается в: изменении объема элементарной ячейки, которое приводит к изменению плотности электростатического заряда, связанного с кристаллической решеткой, что влечет за собой изменение значения ширины запрещенной зоны; расщеплении вырожденных состояний валентной зоны и непрямых минимумов зоны проводимости под действием одноосной компоненты деформации [2]. Кроме того, сдвиги положения краев энергетических зон приводят к изменению значения разрыва валентных зон на гетерогранице. В качестве модельной структуры была взята тонкая псевдоморфно-напряженная КЯ с резкими границами, сформированная на поверхности с ориентацией (110). В таких структурах деформации локализованы в слое узкозонного полупроводника. Расчеты деформаций проведены в рамках макроскопической теории упругости [2].

Участок элементарной ячейки кристалла с кубической симметрией схематично изображен на рис. 1. Обозначим кристаллографические направления [100] как $x$, [010] как $y$ и [001] как $z$. Для определенности будем рассматривать КЯ из материала с большей постоянной решетки, выращенную в матрице из материала с меньшей постоянной решетки. В псевдоморфно-напряженных гетероструктурах с КЯ, выращенных на подложках с ориентацией (110), согласование элементарных ячеек материалов КЯ и матрицы ведет к сжатию материала КЯ в направлениях [001], [11이 и растяжению в направлении [110]. Серыми линиями на рис. 1 показан тот же участок элементарной ячейки после деформации. Деформация КЯ в плоскости гетероструктуры оказывается равна величине рассогласования параметров решеток материалов КЯ и матрицы $f$ :

$$
\varepsilon_{\|}=f
$$

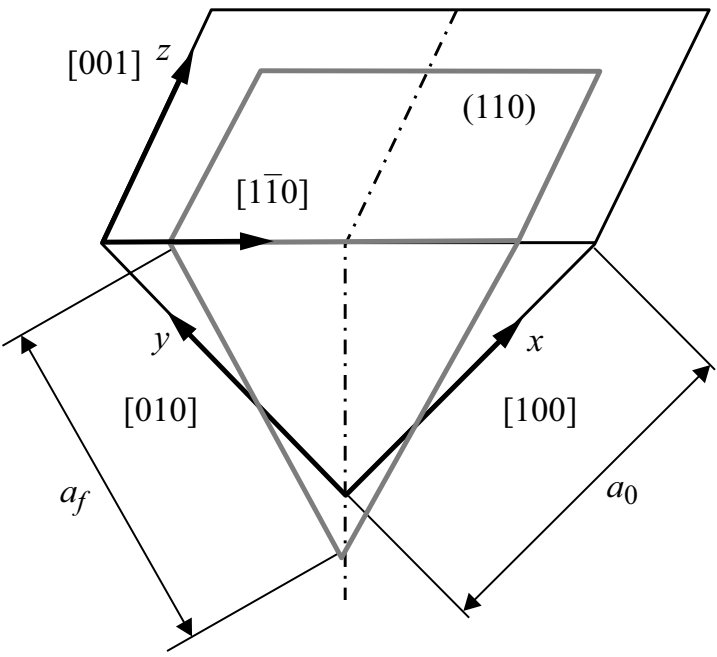

Рис. 1. Схематическое изображение участка элементарной ячейки материала с кубической симметрией, рассеченного плоскостью (110). Серыми линиями изображен этот же участок после деформации в плоскости (110).

В соответствии с результатами [2], деформация КЯ в направлении оси роста составляет

$$
\varepsilon_{\perp}=-D f,
$$

где для структур, выращенных на поверхностях (110), параметр $D$ выражается через упругие константы $c_{11}$, $c_{12}$ и $c_{44}$ как [2]

$$
D=\frac{c_{11}+3 c_{12}-2 c_{44}}{c_{11}+c_{12}+2 c_{44}} .
$$

Компоненты тензора деформаций записываются в виде

$$
\begin{gathered}
\varepsilon_{z z}=\varepsilon_{\|}=f, \\
\varepsilon_{x x}=\varepsilon_{y y} \frac{a_{f}-a_{0}}{a_{0}},
\end{gathered}
$$

где $a_{0}-$ постоянная решетки для ненапряженного материала КЯ, $a_{f}$ - постоянная решетки в направлениях [100] и [010] для напряженного материала, которая определяется выражением

$$
a_{f}^{2}=\frac{a_{0}^{2}}{2}(1-f)^{2}+\frac{a_{0}^{2}}{2}(1+D f)^{2} .
$$

Отсюда компоненты тензора деформации есть

$$
\varepsilon_{x x}=\varepsilon_{y y}=\sqrt{\frac{(1-f)^{2}}{2}+\frac{(1+D f)^{2}}{2}}-1 .
$$

Рассмотрим влияние упругих деформаций на состояния в валентной зоне КЯ. В полупроводниках $\mathrm{A}^{\mathrm{III}} \mathrm{B}^{\mathrm{V}}$ валентная зона состоит из трех подзон - тяжелых, легких и спин-орбитально отщепленных дырок. Гидростатическая упругая деформация, отвечающая за изменение объема элементарной ячейки кристалла и равная

$$
H=\operatorname{Tr}(\varepsilon)=\varepsilon_{x x}+\varepsilon_{y y}+\varepsilon_{z z},
$$


Значения постоянных решетки, упругих констант, ширин запрещенных зон для $\Gamma$-, $X$ - и $L$-долин, деформационных потенциалов и положения потолка валентной зоны $(\mathrm{VBO})$ для материалов $\mathrm{A}^{\mathrm{III}} \mathrm{B}^{\mathrm{V}}$, используемые при расчетах

\begin{tabular}{|c|c|c|c|c|c|c|c|c|c|}
\hline Параметр & $\mathrm{InSb}$ & InAs & InP & $\mathrm{GaSb}$ & GaAs & $\mathrm{GaP}$ & $\mathrm{AlSb}$ & AlAs & AlP \\
\hline$a_{0}, \AA$ & $6.4794^{a}$ & $6.0583^{a}$ & $5.8697^{a}$ & $6.0959^{a}$ & $5.6532^{a}$ & $5.4505^{a}$ & $6.1355^{a}$ & $5.6611^{a}$ & $5.4672^{a}$ \\
\hline$c_{11}$, ГПа & $684.7^{a}$ & $832.9^{a}$ & $1011^{a}$ & $884.2^{a}$ & $1221^{a}$ & $1405^{a}$ & $876.9^{a}$ & $1250^{a}$ & $1330^{a}$ \\
\hline$c_{12}$, ГПа & $373.5^{a}$ & $452.6^{a}$ & $561^{a}$ & $402.6^{a}$ & $566^{a}$ & $620.3^{a}$ & $434.1^{a}$ & $534^{a}$ & $630^{a}$ \\
\hline$c_{44}$, ГПа & $311.1^{a}$ & $395.9^{a}$ & $456^{a}$ & $432.2^{a}$ & $600^{a}$ & $703.3^{a}$ & $407.6^{a}$ & $542^{a}$ & $615^{a}$ \\
\hline$E_{g}^{\Gamma}$, эВ & $0.235^{a}$ & $0.417^{a}$ & $1.4236^{a}$ & $0.812^{a}$ & $1.519^{a}$ & $2.886^{a}$ & $2.386^{a}$ & $3.099^{a}$ & $3.63^{a}$ \\
\hline$E_{g}^{X}, \ni \mathrm{B}$ & $0.63^{a}$ & $1.433^{a}$ & $2.384^{a}$ & $1.141^{a}$ & $1.981^{a}$ & $2.35^{a}$ & $1.696^{a}$ & $2.24^{a}$ & $2.52^{a}$ \\
\hline$E_{g}^{L}$, эB & $0.93^{a}$ & $1.133^{a}$ & $2.014^{a}$ & $0.875^{a}$ & $1.815^{a}$ & $2.72^{a}$ & $2.329^{a}$ & $2.46^{a}$ & $3.57^{a}$ \\
\hline$a_{c}^{\Gamma},{ }^{5} \mathrm{~B}$ & $-6.04^{b}$ & $-3.89^{b}$ & $-6.34^{b}$ & $-9.33^{b}$ & $-9.36^{b}$ & $-9.41^{b}$ & $-8.12^{b}$ & $-7.40^{b}$ & $-6.88^{b}$ \\
\hline$a_{c}^{X}, \ni \mathrm{B}$ & $1.41^{b}$ & $-0.08^{b}$ & $0.59^{b}$ & $-0.20^{b}$ & $-0.16^{b}$ & $0.69^{b}$ & $1.91^{b}$ & $2.54^{b}$ & $3.98^{b}$ \\
\hline$a_{c}^{L}$, э $\mathrm{B}$ & $-2.20^{b}$ & $-3.89^{b}$ & $-3.41^{b}$ & $-4.38^{b}$ & $-4.91^{b}$ & $-4.41^{b}$ & $-2.91^{b}$ & $-3.07^{b}$ & $-1.74^{b}$ \\
\hline$b_{c}^{X}$, эВ & $4.53^{c}$ & $3.7^{c}$ & $3.3^{c}$ & $6.46^{c}$ & $8.61^{e}$ & $5.65^{c}$ & $6.0^{c}$ & $6.11^{c}$ & $6.75^{c}$ \\
\hline$b_{c}^{L}$, э $\mathrm{B}$ & $15.0^{d}$ & $11.35^{e}$ & $11.35^{f}$ & $15.0^{g}$ & $14.26^{e}$ & $11.35^{f}$ & $15.0^{d}$ & $11.35^{f}$ & $11.35^{f}$ \\
\hline$a_{v}$, эB & $0.31^{b}$ & $-1.00^{b}$ & $0.6^{a}$ & $-1.32^{b}$ & $-1.21^{b}$ & $-0.58^{b}$ & $0.73^{b}$ & $1.53^{b}$ & $2.64^{b}$ \\
\hline$b_{v},{ }^{\ni} \mathrm{B}$ & $-2.0^{a}$ & $-1.8^{a}$ & $-2.0^{a}$ & $-2.0^{a}$ & $-2.0^{a}$ & $-1.6^{a}$ & $-1.35^{a}$ & $-2.3^{a}$ & $-1.5^{a}$ \\
\hline$d_{v},{ } \ni \mathrm{B}$ & $-4.7^{a}$ & $-3.6^{a}$ & $-5.0^{a}$ & $-4.7^{a}$ & $-4.8^{a}$ & $-4.6^{a}$ & $-4.3^{a}$ & $-3.4^{a}$ & $-4.6^{a}$ \\
\hline$\Delta_{0}$, эВ & $0.81^{a}$ & $0.39^{a}$ & $0.108^{a}$ & $0.76^{a}$ & $0.341^{a}$ & $0.08^{a}$ & $0.676^{a}$ & $0.28^{a}$ & $0.07^{a}$ \\
\hline VBO, эB & $0^{a}$ & $-0.59^{a}$ & $-0.94^{a}$ & $-0.03^{a}$ & $-0.8^{a}$ & $-1.27^{a}$ & $-0.41^{a}$ & $-1.33^{a}$ & $-1.74^{a}$ \\
\hline
\end{tabular}

Примечание. ${ }^{a}[28],{ }^{b}[29],,^{c}[30],{ }^{d}$ взято значение для GaSb [29,31], ${ }^{e}[2],{ }^{f}$ значение для InAs [2], ${ }^{g}$ [31].

приводит к смещению потолка всех трех подзон валентной зоны

$$
\Delta E_{v}=a_{v} H,
$$

где $a_{v}-$ абсолютный деформационный потенциал для валентной зоны. Одноосная деформация, определяющаяся как

$$
I==\varepsilon_{z z}-\varepsilon_{x x},
$$

приводит к дополнительным смещениям подзон тяжелых $\left(\Delta E_{h h}\right)$, легких $\left(\Delta E_{l h}\right)$ и спин-орбитально отщепленных дырок $\left(\Delta E_{S O}\right)$, определяемым для гетероструктур (110) как собственные значения матрицы $[2,27]$

$$
\left[\begin{array}{ccc}
\frac{\Delta_{0}}{3}-\frac{1}{8}\left(\delta E_{001}+3 \delta E_{111}\right) & -\frac{\sqrt{3}}{8}\left(\delta E_{001}-\delta E_{111}\right) & \frac{\sqrt{6}}{8}\left(\delta E_{001}-\delta E_{111}\right) \\
-\frac{\sqrt{3}}{8}\left(\delta E_{001}-\delta E_{111}\right) & \frac{\Delta_{0}}{3}+\frac{1}{8}\left(\delta E_{001}+3 \delta E_{111}\right) & \frac{\sqrt{2}}{8}\left(\delta E_{001}+3 \delta E_{111}\right) \\
\frac{\sqrt{6}}{8}\left(\delta E_{001}-\delta E_{111}\right) & \frac{\sqrt{2}}{8}\left(\delta E_{001}+3 \delta E_{111}\right) & -\frac{2}{3} \Delta_{0}
\end{array}\right]
$$

Параметры $\delta E_{001}$ и $\delta E_{111}$ есть

$$
\begin{gathered}
\delta E_{001}=2 b_{v} I, \\
\delta E_{111}=\frac{2 \sqrt{3}}{3} d_{v} I,
\end{gathered}
$$

где $v_{v}$ и $d_{v}$ - одноосные деформационные потенциалы для валентной зоны.

Теперь рассмотрим влияние упругих деформаций на состояние в зоне проводимости КЯ. Края зоны проводимости в долинах $\Gamma, X$ и $L$ испытывают смещение под действием гидростатической деформации:

$$
\Delta E_{c}^{\Gamma, X, L}=a_{c}^{\Gamma, X, L} H,
$$

где $a_{c}^{\Gamma, X, L}-$ абсолютные деформационные потенциалы соответственно для долин $\Gamma, X$ и $L$ зоны проводимости.
В ненапряженных полупроводниках $\mathrm{A}^{\mathrm{III}} \mathrm{B}^{\mathrm{V}}$ с кристаллической решеткой, обладающей симметрией сфалерита, электронные состояния вблизи долин $X$ и $L$ зоны проводимости соответственно шестикратно и восьмикратно вырождены. Одноосная деформация материала приводит к снятию вырождения, расщепляя шестикратно вырожденные состояния $X$-долины на двукратно вырожденное состояние $X_{z}$ и четырехкратно вырожденное состояние $X_{x y}$ и расщепляя восьмикратно вырожденные состояния $L$-долины на четырехкратно вырожденные состояния $L^{111,11 \overline{1}}$ и $L^{\overline{1} 11,1 \overline{1} 1}$. Величины смещения краев подзон определяется как

$$
\begin{gathered}
\Delta E_{c}^{x y}=-\frac{1}{3} b_{c}^{X} I, \\
\Delta E_{c}^{z}=\frac{2}{3} b_{c}^{X}, \\
\Delta E_{c}^{111,11 \overline{1}}=\frac{2}{9} b_{c}^{L} I, \\
\Delta E_{c}^{\overline{1} 11,1 \overline{1} 1}=-\frac{2}{9} b_{c}^{L} I,
\end{gathered}
$$

где $b_{c}^{X}$ и $b_{c}^{L}$ - одноосные деформационные потенциалы для $X$ - и $L$-долин соответственно. Отметим, что отличительной особенностью энергетического спектра гетероструктур, выращенных на подложках с ориентацией (110), является расщепление обеих непрямых долин $X$ и $L$ зоны проводимости на $X_{z}, X_{x y}$ и $L^{111,11 \overline{1}}, L^{\overline{1} 11,1 \overline{1} 1}$ соответственно, в то время как в гетероструктурах (001) расщеплена только $X$-долина, а в гетероструктуpax (111) - только $L$-долина. При расчетах использовались параметры материалов, представленные в таблице.

К сожалению, наряду с достоверно определенными параметрами соединений $\mathrm{A}^{\mathrm{III}} \mathrm{B}^{\mathrm{V}}$, такими как постоянные 

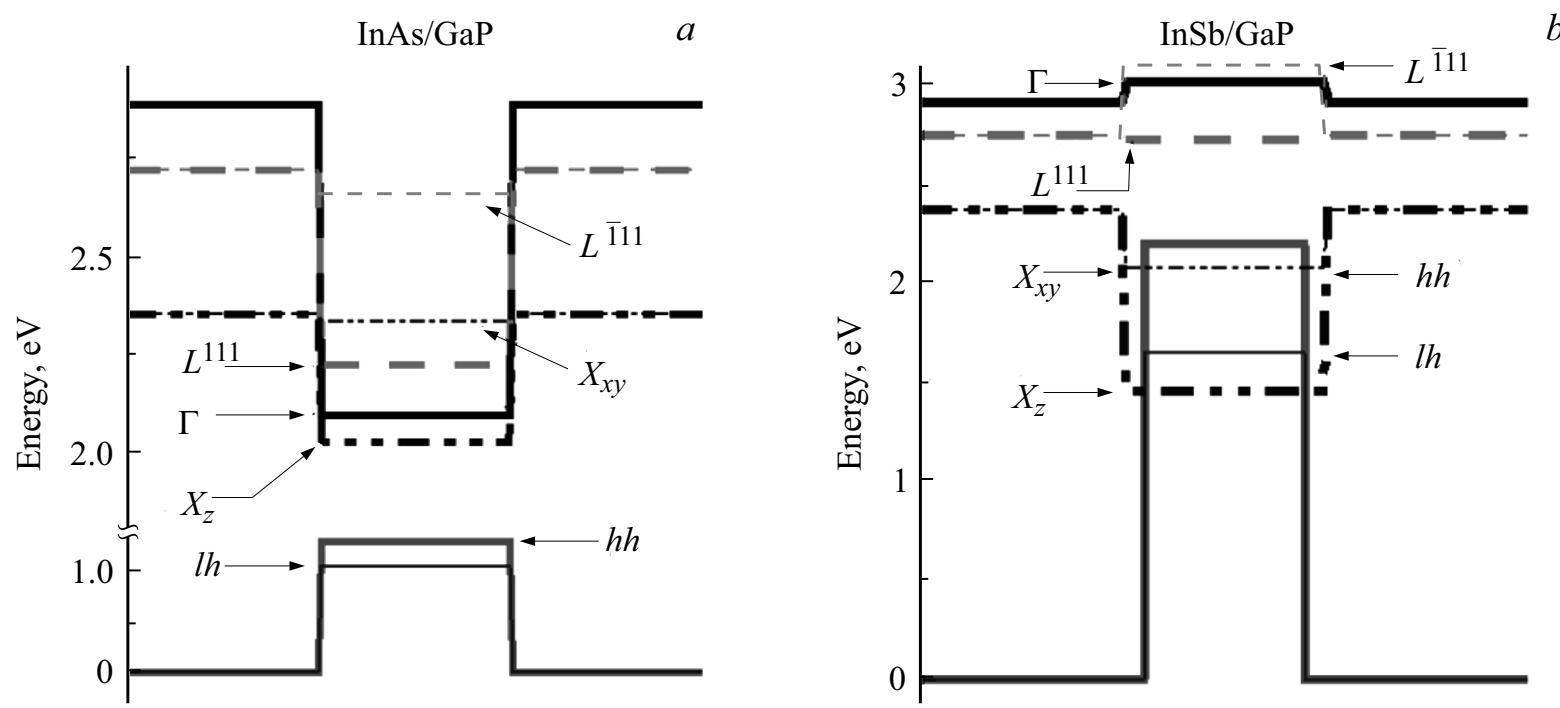

Рис. 2. Зонные диаграммы, рассчитанные для гетероструктур на подложках с ориентацией (110): $a-\mathrm{InAs} / \mathrm{GaP}, b-\mathrm{InSb} / \mathrm{GaP}$. $b$ : ширина КЯ в валентной зоне уменьшена для облегчения восприятия. Электронные и дырочные состояния в Г-точке зоны Бриллюэна - сплошные линии. Состояния электронов в $L$ - и $X$ - долинах зоны проводимости - штриховые и штрихпунктирные линии соответственно. Стрелками показаны положения краев энергетических зон в КЯ.

решетки, упругие постоянные и деформационные потенциалы, имеются параметры, значения которых плохо определены. Фактически, положения потолка валентной зоны для материалов $\mathrm{A}^{\mathrm{III}} \mathrm{B}^{\mathrm{V}}$ известны с точностью \pm 50 мэВ [29], что накладывает ограничения на определение рода гетероструктуры посредством расчетов.

Уровни размерного квантования носителей заряда рассчитывались в однозонном приближении. Согласно результатам работ $[32,33]$, метод эффективной массы применим для расчета энергетического спектра гетероструктур с размерами порядка единиц нанометров, когда слой КЯ вмещает несколько постоянных решетки. Для упрощения расчета мы не принимали во внимание кулоновское взаимодействие электронов и дырок (экситонный эффект). Также не принимались во внимание эффекты интерфейсного смешивания электронных и дырочных состояний.

\section{3. Результаты и обсуждение}

Расчеты позволяют разделить рассматриваемые гетероструктуры на следующие категории: 1) прямозонные структуры второго и первого рода, не представляющие интереса для нашего исследования; 2) структуры первого рода с непрямой запрещенной зоной; наконец, 3) непрямозонные структуры, для которых неопределенность значений используемых в расчетах параметров не позволяет однозначно определить род энергетического спектра.

Рассмотрим интересующие нас категории гетероструктур подробнее. Вторую категорию гетероструктур, в свою очередь, можно разделить на структуры, для которых энергетический спектр (А) не зависит от геометрических размеров (для модельной структуры от толщины КЯ), или (В) зависит от толщины КЯ. К структурам типа (A) относятся КЯ InSb в матрицах $\mathrm{GaSb}, \mathrm{GaAs}, \mathrm{GaP}, \mathrm{AlSb}, \mathrm{AlAs}$ и $\mathrm{AlP}, \mathrm{InAs} / \mathrm{GaP}$ и $\mathrm{AlSb} / \mathrm{GaP}$, а к структурам типа (B) КЯ InAs/AlAs и GaSb/AlSb.

Энергетические диаграммы типичных структур типа (А), рассчитанные для КЯ $\mathrm{InAs} / \mathrm{GaP}$ и $\mathrm{InSb} / \mathrm{GaP}$, представлены на рис. $3, a, b$ соответственно. Основное электронное состояние в КЯ InAs/GaP принадлежит двукратно вырожденной $X_{z}$-долине зоны проводимости InAs, а дырочное состояние - подзоне тяжелых дырок InAs. Возбужденные электронные и дырочные состояния лежат в $\Gamma-, L^{111,11 \overline{1}}$ - и $X_{x y}$-долинах зоны проводимости и в подзоне легких дырок соответственно. Схожее строение имеют КЯ $\mathrm{AlSb} / \mathrm{GaP}, \mathrm{InSb} / \mathrm{GaSb}$ и InSb/AlSb: в них также основное электронное состояние принадлежит $X_{z}$-подзоне зоны проводимости, а основное дырочное подзоне тяжелых дырок. В КЯ $\mathrm{InSb} / \mathrm{GaSb}$ и $\mathrm{InSb} / \mathrm{AlSb}$ возбужденные состояния образуются электронами из Г-долины зоны проводимости.

Основное электронное состояние в КЯ $\mathrm{InSb} / \mathrm{GaP}$, как видно из рис. $2, b$, принадлежит двукратно вырожденной $X_{z}$-долине зоны проводимости $\mathrm{InSb}$, а дырочное состояние - подзоне тяжелых дырок InSb. Возбужденные электронные и дырочные состояния лежат в $X_{x y}$-долине зоны проводимости и подзоне легких дырок соответственно. Энергетическое строение гетероструктур $\mathrm{InSb} / \mathrm{GaP}$ примечательно перекрытием состояний $X$-долины зоны проводимости InSb и валентной зоны InSb, что может привести к формированию инвертированного энергетического спектра. Аналогичное энергетическое строение с перекрытием электронных и дырочных зон имеют КЯ $\mathrm{InSb} / \mathrm{GaAs}, \mathrm{InSb} / \mathrm{AlAs}$ и InSb/AlP. 
$a$

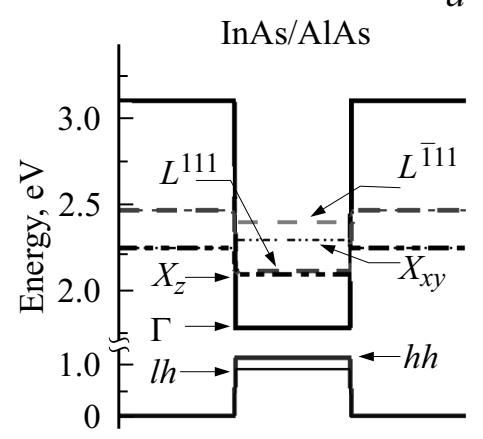

$b$

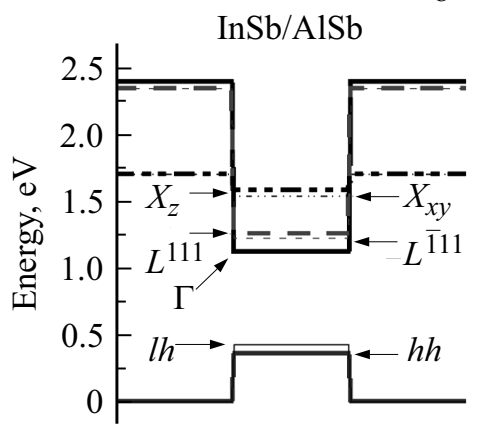

$c$

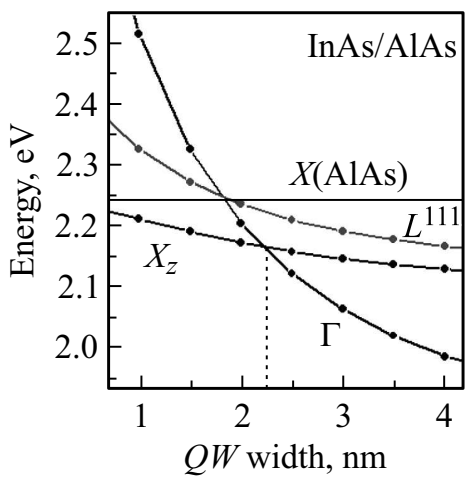

Рис. 3. Зонные диаграммы, рассчитанные для гетероструктур на подложках с ориентацией (110): $a-\mathrm{InAs} / \mathrm{AlAs}, b-\mathrm{GaSb} / \mathrm{AlSb}$. Электронные и дырочные состояния в Г-точке зоны Бриллюэна - сплошные линии. Состояния электронов в $L$ - и $X$-долинах зоны проводимости - штриховые и штрихпунктирные линии соответственно. Стрелками показаны положения краев энергетических зон в КЯ. $c$ - зависимости положения уровней размерного квантования электронов в долинах $Г, X_{Z}, L^{111}, L^{11 \overline{1}}$ зоны проводимости InAs от толщины KЯ (QW) InAs/AlAs; горизонтальная линия соответствует положению дна зоны проводимости в матрице AlAs; вертикальная пунктирная линия - критическая ширина КЯ, при которой происходит смена энергетического строения с прямозонного на непрямозонное.

Изученные нами ранее непрямозонные гетероструктуры, выращенные на подложках с ориентацией (001), имеют основное электронное состояние, лежащее в четырехкратно вырожденной $X_{x y}$-долине зоны проводимости $[16,17,19,21-23,25]$. Различия энергетического спектра гетероструктур, выращенных на подложках с ориентацией (001) и (110), обусловлены изменением распределения деформаций в кристаллической решетке КЯ. При сжатии материала КЯ в плоскости (110) уменьшается энергия электронов $X_{z}$-долины, квазиимпульс которых направлен вдоль кристаллографического направления [001], в то время как при сжатии КЯ в плоскости (001) снижается энергия электронов $X_{x y}$-долины, квазиимпульс которых направлен вдоль кристаллографических направлений [100] и [010] [2].

Энергетические диаграммы, рассчитанные для гетероструктур типа (B) - КЯ InAs/AlAs и GaSb/AlSb, представлены на рис. 3, $a, b$ соответственно. Наименьшую энергию в зоне проводимости КЯ InAs/AlAs имеет дно Г-долины, а возбужденные состояния принадлежат $X_{z}$ - и $L^{111,11 \overline{1}}$-долинам зоны проводимости InAs (см. рис. 3,a). Энергия уровня размерного квантования для ,легких“ электронов в Г-долине увеличивается с уменьшением толщины КЯ быстрее, чем для „тяжелых“ электронов в $X_{z}$ - и $L^{111,11 \overline{1}}$-долинах зоны проводимости, поэтому при критической толщине КЯ $(\sim 2.2 \mathrm{HM})$, как это видно из рис. 3, $c$, происходит смена энергетического спектра КЯ с прямозонного на непрямозонный. Основное дырочное состояние лежит в подзоне тяжелых дырок независимо от размеров КЯ.

Гетеросистема GaSb/AlSb, зонная диаграмма которой показана на рис. 3, $b$, характеризуется относительно небольшим $(0.65 \%)$ и положительным по знаку рассогласованием параметров решеток, т.е. слой $\mathrm{GaSb}$ ока- зывается растянутым в плоскости (110). Наименьшую энергию в зоне проводимости КЯ $\mathrm{GaSb} / \mathrm{AlSb}$ имеет состояние электронов в Г-долине зоны проводимости $\mathrm{GaSb}$. Возбужденные электронные состояния, принадлежащие долинам $L^{\overline{1} 11,1 \overline{1} 1}, L^{111,11 \overline{1}}, X_{x y}$ и $X_{z}$ зоны проводимости $\mathrm{GaSb}$, расположены ниже дна зоны проводимости матрицы AlSb. Наибольшую энергию в валентной зоне имеет потолок подзоны легких дырок. Так же, как и для КЯ InAs/AlAs, изменение ширины КЯ $\mathrm{GaSb} / \mathrm{AlSb}$ может привести к смене энергетического строения КЯ с прямозонного на непрямозонное, равно как и к переходу основного дырочного состояния КЯ GaSb/AlSb из подзоны легких дырок в подзону тяжелых дырок.

К третьей категории гетероструктур мы отнесли структуры, для которых неопределенность значений используемых в расчетах параметров не позволяют однозначно определить род энергетического спектра. Энергетическая диаграмма, рассчитанная для типичной гетероструктуры из этой категории (КЯ AlAs/AlP) представлена на рис. 4. Наименьшую энергию в слое AlAs, как видно из рисунка, имеют состояния $X_{z}$-долины зоны проводимости, лежащие на 30 мэВ ниже дна зоны проводимости матрицы AlP. Состояния электронов в долинах $X_{x y}, L^{111,11 \overline{1}}, L^{\overline{1} 11,1 \overline{1} 1}$ и Г зоны проводимости AlAs лежат выше дна зоны проводимости AlP. Основное дырочное состояние принадлежит подзоне тяжелых дырок. Как уже обсуждалось выше, в разд. 2, положение потолков валентных зон полупроводников $\mathrm{A}^{\mathrm{III}} \mathrm{B}^{\mathrm{V}}$ известно с точностью \pm 50 мэВ [29]. Это приводит к неопределенности в величине разрыва валентных зон для пары материалов \pm 100 мэВ, что превосходит расчетное значение величины энергетического барьера в зоне проводимости для КЯ AlAs/AlP. Следовательно, основное электронное состояние может лежать как в слое AlAs, так и в матрице AlP, 


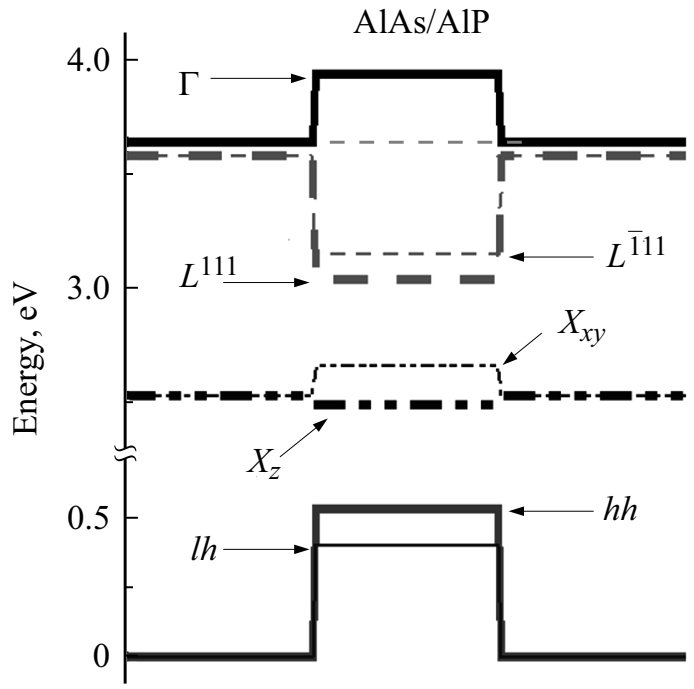

Рис. 4. Зонная диаграмма, рассчитанная для гетероструктуры AlAs/AlP на подложке с ориентацией (110). Электронные и дырочные состояния в Г-точке зоны Бриллюэна - сплошные линии. Состояния электронов в $L$ - и $X$-долинах зоны проводимости - штриховые и штрихпунктирные линии соответственно. Стрелками показаны положения краев энергетических зон в КЯ.

а значит, проведенные расчеты не позволяют однозначно отнести КЯ AlAs/AlP к системам с энергетическим спектром первого либо второго рода. Уверенно можно лишь утверждать, что основное электронное состояние KЯ AlAs/AlP принадлежит $X$-долине зоны проводимости. Ситуация аналогична для гетероструктур InAs/AlP, $\mathrm{AlSb} / \mathrm{AlP}, \mathrm{GaSb} / \mathrm{GaP}, \mathrm{GaAs} / \mathrm{GaP}$ и AlSb/AlAs.

Результаты расчетов можно обобщить следующим образом.

- Зонные диаграммы для гетероструктур InAs/GaP, $\mathrm{AlSb} / \mathrm{GaP}$, и $\mathrm{InSb}$, сформированных в матрицах $\mathrm{GaSb}$, $\mathrm{GaAs}, \mathrm{GaP}, \mathrm{AlSb}, \mathrm{AlAs}$ и AlP, не зависят от геометрических параметров КЯ и КТ. В этих гетеросистемах реализуется энергетический спектр первого рода с непрямой запрещенной зоной и основным электронным состоянием, принадлежащим $X_{z}$-долине зоны проводимости.

- Энергетический спектр гетероструктур InAs/AlAs и $\mathrm{GaSb} / \mathrm{AlSb}$, напротив, определяется геометрическими параметрами. В „широких“ КЯ основное электронное состояние принадлежит Г-долине, а в „узких“ $-X$ или $L$-долине зоны проводимости InAs или $\mathrm{GaSb}$ соответственно.

- Однозначно отнести КЯ AlAs/AlP, InAs/AlP, $\mathrm{AlSb} / \mathrm{AlP}, \mathrm{GaSb} / \mathrm{GaP}, \mathrm{GaAs} / \mathrm{GaP}$, и AlSb/AlAs к структурам первого либо второго рода не представляется возможным. Основное электронное состояние в этих гетероструктурах принадлежит $X$-долине зоны проводимости и может быть локализовано как в материале КЯ, так и в матрице.

Таким образом, формирование энергетического спектра первого рода с непрямой запрещенной зоной в гетероструктурах на основе соединений $\mathrm{A}^{\mathrm{III}} \mathrm{B}^{\mathrm{V}}$ на подложках ориентации (110) является скорее правилом, чем исключением.

Гетероструктуры первого рода с непрямой зоной проводимости могут быть удобными объектами для анализа физических процессов, изучение которых в других типах гетероструктур затруднено. Характерным примером такого процесса является спиновая динамика локализованных экситонов. Сильная локализация экситонов приводит к подавлению механизмов, определяющих переворот спина свободных квазичастиц, поэтому время жизни спиновой поляризации экситонов становится заметно больше, чем время жизни экситона в прямозонных гетероструктурах [34,35]. Ориентация (110) обеспечивает дополнительное подавление механизмов переворота спина в гетероструктурах, что в совокупности с длительным временем жизни экситона, обусловленным законом сохранения квазиимпульса, делает непрямозонные КТ и КЯ первого рода интересными объектами для изучения спиновой динамики экситонов.

Для изучения спиновой динамики спиновую систему необходимо вывести из равновесия [36]. Неоднородность спиновой населенности можно создавать с помощью оптической ориентации - за счет возбуждения циркулярно поляризованным светом. Это - хорошо отработанная и активно применяемая методика при изучении спиновой динамики экситонов и трионов в прямозонных полупроводниках, КЯ и КТ [36,37]. Из-за слабости экситон-фотонного взаимодействия оптическая ориентация неприменима для возбуждения спиновой подсистемы непрямозонных структур. Поэтому в общем случае для изучения спиновой динамики экситонов в таких гетероструктурах измеряется динамика наведенной магнитным полем циркулярной поляризации фотолюминесценции (ФЛ) [38-40]. Однако в непрямозонных гетероструктурах с возбужденным электронным состоянием, принадлежащим Г-долине зоны проводимости, появляется возможность использования оптической ориентации для изучения спиновой динамики экситонов в режиме квазирезонансного возбуждения ФЛ [41]. В КЯ и КТ на основе соединений $\mathrm{A}^{\mathrm{III}} \mathrm{B}^{\mathrm{V}}$ на подложках ориентации (110) возбужденные электронные состояния в Г-долине зоны проводимости формируются в гетеросистемах InAs/GaP, InSb/GaSb, InSb/AlSb, InAs/AlAs и $\mathrm{GaSb} / \mathrm{A} 1 \mathrm{Sb}$.

Рассматривая возможности получения описанных выше КЯ и КТ, необходимо отметить, что наиболее интересными являются гетеросистемы, формирующиеся на доступных подложках GaAs, согласованных по параметру решетки с материалом матрицы: $\mathrm{InSb} / \mathrm{GaAs}$, InSb/AlAs, InAs/AlAs и AlSb/AlAs. Из них наиболее перспективными для эпитаксиального роста представляются гетероструктуры InAs/AlAs. Поскольку, с одной стороны, пара материалов InAs и AlAs характеризуется заметно меньшим рассогласованием параметров решетки (6.5\%), по сравнению с гетероструктурами на основе InSb/GaAs, InSb/AlAs (12.6\%) и AlSb/AlAs 
(7.7\%) [28], а с другой стороны, формирование гетероструктур InSb/GaAs, InSb/AlAs и AlSb/AlAs затруднено из-за существенного различия в оптимальных условиях эпитаксиального роста антимонидов и арсенидов соединений $\mathrm{A}^{\mathrm{III}} \mathrm{B}^{\mathrm{V}}$. Для получения слоев GaAs и $\mathrm{AlAs}$ c минимальной концентрацией структурных и точечных дефектов методом молекулярно-лучевой эпитаксии оптимальной считается температура $600-650^{\circ} \mathrm{C}$ [42], в то время как для роста слоев InSb и AlSb оптимальной является температура $300-350^{\circ} \mathrm{C}$ [43].

\section{4. Заключение}

Показано, что формирование гетероструктур первого рода с непрямой запрещенной зоной на подложках с ориентацией (110) типично для бинарных полупроводниковых соединений $\mathrm{A}^{\mathrm{III}} \mathrm{B}^{\mathrm{V}}$. Сочетание длительного времени жизни экситонов, обусловленного правилами отбора, и устойчивых спиновых состояний, обусловленных кристаллографической ориентаций, открывает перспективы для использования таких гетероструктур в качестве модельных объектов при изучении спиновой динамики экситонов и потенциально как базы для создания спиновых устройств передачи, обработки и хранения информации.

Работа выполнена при финансовой поддержке в рамках постановления № 211 правительства Российской Федерации, контракт № 02.А03.21.0006, РФФИ (проекты № 16-32-60015 мол_а_дк, № 19-02-00098 и № 19-52-12001).

\section{Список литературы}

[1] Z.M. Wang. Self Assembled Quantum Dots (Springer, N.Y., 2008).

[2] C.G. Van de Walle. Phys. Rev. B, 39, 1871 (1989).

[3] G. Wang, B.L. Liu, A. Balocchi, P. Renucci, C.R. Zhu, T. Amand, C. Fontaine, X. Marie. Nature Commun., 4, 2372 (2013).

[4] A. Hernandez-Minguez, K. Biermann, R. Hey, P.V. Santos. Phys. Rev. B, 94, 125311 (2016).

[5] K.F. Karlsson, M.A. Dupertuis, D.Y. Oberli, E. Pelucchi, A. Rudra, P.O. Holtz, E. Kapon. Phys. Rev. B, 81, 161307 (2010).

[6] A. Schliwa, M. Winkelnkemper, A. Lochmann, E. Stock, D. Bimberg. Phys. Rev. B, 80, 161307(R) (2009).

[7] Y.S. Chen, S. Falt, W. Wegscheider, G. Salis. Phys. Rev. B, 90, 121304(R) (2014).

[8] M. Dyakonov, V. Perel. Sov. Phys. Solid State, 13, 3023 (1972).

[9] O.D.D. Couto, jr., F. Iikawa, J. Rudolph, R. Hey, P.V. Santos. Phys. Rev. Lett., 98, 036603 (2007),

[10] M. Kohda, G. Salis. Semicond. Sci. Technol., 32 (7), 073002 (2017).

[11] T.S. Shamirzaev, J. Debus, D.S. Abramkin, D. Dunker, D.R. Yakovlev, D.V. Dmitriev, A.K. Gutakovskii, L.S. Braginsky, K.S. Zhuravlev, M. Bayer. Phys. Rev. B, 84, 155318 (2011).
[12] T.S. Shamirzaev, J. Debus, D.R. Yakovlev, M.M. Glazov, E.L. Ivchenko, M. Bayer. Phys. Rev. B, 94, 045411 (2016).

[13] W. Tomm, T. Elsaesser, Yu.I. Mazur, H. Kissel, G.G. Tarasov, Z.Ya. Zhuchenko, W.T. Masselink. Phys. Rev. B, 67, 045326 (2003).

[14] Т.С. Шамирзаев. ФТП, 45, 97 (2011).

[15] M.-E. Pistol, C.E. Pryor. Phys. Rev. B, 80, 035316 (2009).

[16] T.S. Shamirzaev, A.V. Nenashev, A.K. Gutakovskii, A.K. Kalagin, K.S. Zhuravlev, M. Larsson, P.O. Holtz. Phys. Rev. B, 78, 085323 (2008).

[17] T.S. Shamirzaev, A.M. Gilinsky, A.K. Kalagin, A.V. Nenashev, K.S. Zhuravlev. Phys. Rev. B, 76, 155309 (2007).

[18] T.S. Shamirzaev, D.S. Abramkin, A.K. Gutakovskii, M.A. Putyato. Appl. Phys. Lett., 97, 023108 (2010).

[19] D.S. Abramkin, M.A. Putyato, S.A. Budennyy, A.K. Gutakovskii, B.R. Semyagin, V.V. Preobrazhenskii, O.F. Kolomys, V.V. Strelchuk, T.S. Shamirzaev. J. Appl. Phys., 112, 083713 (2012).

[20] Д.С. Абрамкин, М.А. Путято, А.К. Гутаковский, Б.Р. Семягин, В.В. Преображенский, Т.С. Шамирзаев. ФТП, 46, 1571 (2012).

[21] T.S. Shamirzaev, D.S. Abramkin, A.K. Gutakovskii, M.A. Putyato. Письма ЖЭТФ, 95, 601 (2012).

[22] D.S. Abramkin, V.T. Shamirzaev, M.A. Putyato, A.K. Gutakovskii, T.S. Shamirzaev. Письма ЖЭТФ, 99, 81 (2014).

[23] Д.С. Абрамкин, К.М. Румынин, А.К. Бакаров, Д.А. Колотовкина, А.К. Гутаковский, Т.С. Шамирзаев. Письма ЖЭТФ, 103, 785 (2016).

[24] Д.С. Абрамкин, А.К. Бакаров, М.А. Путято, Е.А. Емельянов, Д.А. Колотовкина, А.К. Гутаковский, Т.С. Шамирзаев. ФТП, 51, 1282 (2017).

[25] Д.С. Абрамкин, А.К. Бакаров, А.К. Гутаковский, Т.С. Шамирзаев. ФТП, 52, 1280 (2018).

[26] D. Bimberg, M. Grundmann, N.N. Ledentsov. Quantum Dot Heterostructures (N.Y., Wiley, 1999).

[27] F.H. Pollak, M. Cardona. Phys. Rev., 172, 816 (1968).

[28] I. Vurgaftman, J.R. Meyer, L.R. Ram-Mohan. J. Appl. Phys., 89, 5815 (2001).

[29] S.H. Wei, A. Zunger. Appl. Phys. Lett., 72, 2011 (1998).

[30] M.C. Munoz, G. Armelles. Phys. Rev. B, 48, 2839 (1993).

[31] Landolt-Bornstein Numerical Data and Functional Relationships in Scince and Technology, ed. by O. Madelung, M. Schultz, H. Weiss. New series, vol. 17 (1982) [Semiconductors-Basic Data, 2nd edn, ed. by O. Madelung (Springer, N.Y., 1996)].

[32] Al.L. Efros, M. Rosen, M. Kuno, M. Nirmal, D.J. Norris, M. Bawendi. Phys. Rev. B, 54, 4843 (1996).

[33] M.G. Burt. J. Phys.: Condens. Matter, 4, 6651 (1992).

[34] M. Paillard, X. Marie, P. Renucci, T. Amand, A. Jbeli, J.M. Gérard. Phys. Rev. Lett., 86, 1634 (2001).

[35] P. Nahálková, D. Sprinzl, P. Malý, P. Nĕmec, V.N. Gladilin, J.T. Devreese. Phys. Rev. B, 75, 113306 (2007).

[36] M.W. Wu, J.H. Jiang, M.Q. Weng. Phys. Reports, 493, 61 (2010).

[37] R. Ohta, H. Okamoto, T. Tawara, H. Gotoh, H. Yamaguchi. Phys. Rev. Lett., 120, 267401 (2018).

[38] T.S. Shamirzaev, J. Rautert, D.R. Yakovlev, J. Debus, A.Yu. Gornov, M.M. Glazov, E.L. Ivchenko, M. Bayer. Phys. Rev. B, 96, 035302 (2017).

[39] Т.С. Шамирзаев. ФТТ, 60, 1542 (2018).

[40] Е.Л. Ивченко. ФТТ, 60, 1503 (2018). 
[41] J. Debus, T.S. Shamirzaev, D. Dunker, V.F. Sapega, E.L. Ivchenko, D.R. Yakovlev, A.I. Toropov, M. Bayer. Phys. Rev. B, 90, 125431 (2014).

[42] P.M. Petroff, A.C. Gossard, W. Wiegmann. Appl. Phys. Lett., 45, 620 (1984)

[43] E. Michel, G. Singh, S. Slivken, C. Besikci, P. Bove, I. Ferguson, M. Razegh. Appl. Phys. Lett., 65, 3338 (1994).

Редактор Л.В. Шаронова

\section{Semiconductor heterostructures of type I and indirect bandgap formed on substrates with (110) orientation}

D.S. Abramkin ${ }^{1,2}$, T.S. Shamirzaev ${ }^{1,2,3}$

${ }^{1}$ Rzhanov Institute of Semiconductor Physics, Siberian Branch of Russian Academy of Sciences, 630090 Novosibirsk, Russia

${ }^{2}$ Novosibirsk State University, 630090 Novosibirsk, Russia

${ }^{3}$ Ural Federal University,

620002 Yekaterinburg, Russia

Abstract Type I heterostructures and indirect bandgap are interesting objects for localized exciton spin dynamics investigations, which are complicated in other types of heterostructures. It is demonstrated, that structures with this type of band alignment can be formed from binary III-V materials on substrates with (110) orientation. The effect of elastic strain distribution and the structure of conduction band in reciprocal space on energy spectrum of heterostructures is discussed. 